\title{
Aortoenteric Fistula-An Uncommon Cause of Gastrointestinal Bleeding. A Review of the Literature
}

ISSN: 2637-7632

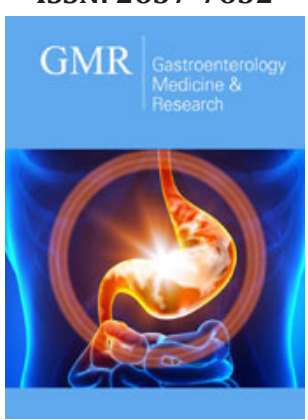

${ }^{* 1}$ Corresponding author: Antoine Abi Abboud, Department of Gastroenterology and Hepatology, Faculty of Medical Sciences, Lebanese University, Lebanon

Submission: 侮 March 12, 2019

Published: : March 29, 2019

Volume 3 - Issue 1

How to cite this article: Antoine Ab A, Julien S, Joseph H, Setrida G Aortoenteric Fistula-An Uncommon Cause of Gastrointestinal Bleeding. A Review of the Literature. Gastro Med Res. 3(1) GMR.000553. 2019.

DOI: 10.31031/GMR.2019.03.000553

Copyright@ Antoine Abi Abboud, This article is distributed under the terms of the Creative Commons Attribution 4.0 International License, which permits unrestricted use and redistribution provided that the original author and source are credited.

\author{
Antoine Abi Abboud ${ }^{1,2 *}$, Julien Sfeir ${ }^{2,3}$, Joseph Haddad ${ }^{3}$ and Setrida Gemayel ${ }^{2,4}$ \\ ${ }^{1}$ Department of Gastroenterology and Hepatology, Lebanon \\ ${ }^{2}$ Faculty of Medical Sciences, Lebanon \\ ${ }^{3}$ Department of Thoracic and Vascular Surgery, Lebanon \\ ${ }^{4}$ Department of Internal Medicine, Lebanon
}

\begin{abstract}
Aortoenteric fistula is a pathological communication between the aorta and any portion of the gastrointestinal tract. It is a rare cause of upper or lower gastrointestinal bleeding. Clinical presentation may vary from a "herald" bleed to a life-threatening deadly bleeding if left untreated. Primary aortoenteric fistula is usually caused by an untreated abdominal aortic aneurysm, whereas secondary aortoenteric fistula occurs as a complication of an abdominal aortic aneurysm repair. Diagnosis is often difficult and delayed. Initial diagnostic tests may include computerized tomography scan and esophagogastroduodenoscopy. Urgent surgery is still the recommended treatment. In this article, we aim to provide a comprehensive update on the type, diagnosis, management, and prognosis of aortoenteric fistulas.
\end{abstract}

Keywords: Aortoenteric fistula; Abdominal aortic aneurysm; Gastrointestinal bleed

Abbreviations: AEF: Aortoenteric Fistula; PAEF: Primary Aorto enteric Fistula; SAEF: Secondary Aorto Enteric Fistula; AAA: Abdominal Aortic Aneurysm; GI: Gastro Intestinal; EGD: Esophago Gastro Duodenoscopy

\section{Introduction}

Aortoenteric fistulas (AEFs) are abnormal connections between the aorta and gastrointestinal (GI) tract, first described by Sir Astley Cooper in the early 19th century. Primary aortoenteric fistulas (PAEFs) are rare with an incidence rate of $0.1 \%$ to $0.8 \%$ out of AEFs. They are associated mainly with a pre-existing abdominal aortic aneurysm (AAA), in $85 \%$ of cases. Other causes such as infections, syphilis, tuberculosis, peptic ulcer disease, malignancy and radiotherapy have also been described. Secondary aortoenteric fistulas (SAEFs) are more common, in $0.4-2.4 \%$ of cases, and result as a complication of a AAA repair. Aortoenteric fistulas are associated with a high rate of mortality (65-100\%). Most of them occur in the duodenum (83\%), mainly in the 3rd and 4th portion, although they may occasionally occur in other segments of GI tract, such as the small intestine, colon or even appendix [1-5].

\section{Discussion}

Fistula formation between the aorta and the intestinal tract was first described in 1829 by English surgeon Sir Astley Cooper. He observed a lethal communication between the aorta and gastrointestinal tract causing massive upper gastrointestinal bleeding. Two types are described: Primary and Secondary AEFs [6-10].

\section{PAEF}

Primary Aortoenteric fistula is defined as a direct spontaneous communication between the aorta and the gastro-intestinal lumen without history of surgery or graft. It represents a rare and severe complication of the abdominal aortic aneurysm (AAA). The primary form, 
less common with an incidence of 0.1 to $0.8 \%$, is due to the erosion of the intestinal wall by the aortic aneurysm in the presence of predisposing factors such as atherosclerosis (60-80\% of cases), infections (e.g., syphilis and tuberculosis), collagen diseases, cancer (lymphoma) or mechanical stress (e.g., biliary calculi, ulcers, or radiation) (Figure 1).

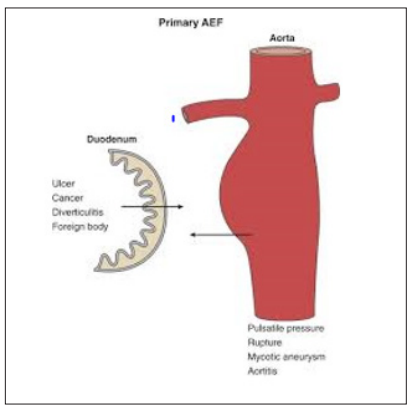

Figure 1:

\section{SAEF}

Secondary Aortoenteric fistula, described for the first time by Brock in 1953, two years after the first abdominal aortic homograft implementation due to aneurysm, occurs as a complication of a AAA repair. In most cases, the portal of entry is the suture line. In other cases, the fistula is caused by the infection of the prosthetic graft, due to colonization of the graft by skin flora at time of surgery (Staphylococcus epidermidis), or due to the contamination of the graft by diverse microbes including Gram-positive organisms, Gramnegative organisms, and anaerobes coming from the bowel flora (Enterobacter, E. coli, Pseudomonas, Klebsiella...). In some cases, after repair, the aortic pulsations may form a pseudoaneurysm that may produce shear stress on the GI mucosa, resulting in erosion and fistula (Figure 2).

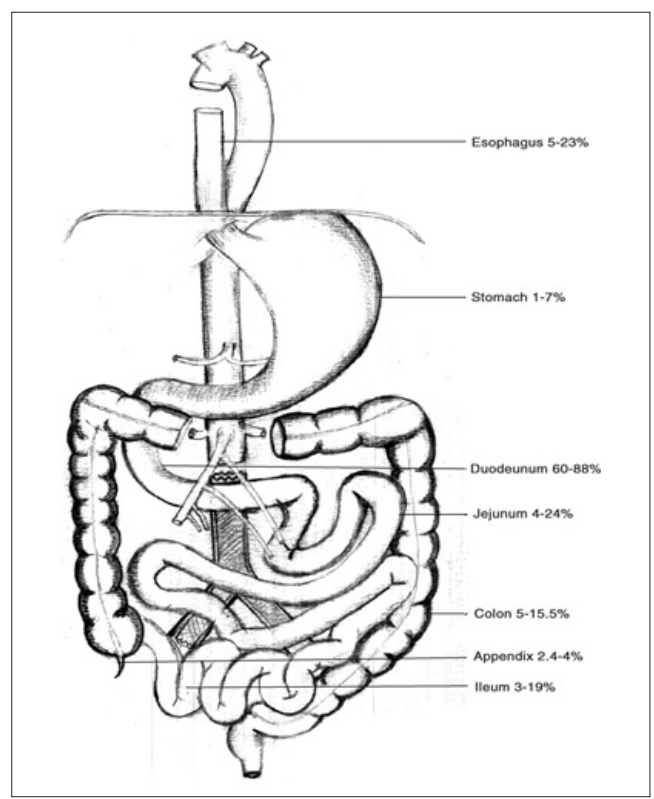

Figure2: The incidence of secondary aortoenteric fistula at various anatomical locations.

\section{Epidemiology}

The abdominal aorta is more likely to be involved than the thoracic aorta ( 56 vs. $44 \%$ ). In one-third of the cases, the abdominal aorta forms a channel with the duodenum. This is secondary to the anatomical position of the third part of the duodenum, which lies between the superior mesenteric artery and the abdominal aorta. The jejunum and ileum are only affected in $4 \%$ of cases. Rarely, it may involve esophagus, ascending, transverse, sigmoid colon, or rectum. In untreated patients or in patients having active bleeding, the mortality is extremely high (95-100\%). It is reduced to around $30-40 \%$ in patients treated with surgery. Epidemiological data reveal that the average age of diagnosed PAEF is 64-years-old and that it mainly affects males more than females with a 3:1 ratio. About $83 \%$ of PAEF is associated with a fusiform AAA having a mean diameter $6.2 \mathrm{~cm}$ [11-17].

\section{Signs and Symptoms}

The most common clinical presentation of PAEF and SAEF is upper or lower GI bleed, which may vary in its presentation from "herald" bleeding to overt hemorrhagic shock. Herald bleeding is an episode of transient or self-limiting hemorrhage. Formation of a clot in the fistula will stop bleeding but infusion therapy, or increasing blood pressure will lead to hemorrhage again. Although, after GI bleed, sepsis or fever is the commonest presentation in patients with SAEF, PAEF can be associated with episodes of hematemesis and melena (64\%), abdominal pain (32\%) and the palpation of an abdominal pulsating mass (25\%), and it is rarely related to signs sepsis (fever, leukocytosis). The classic triad of abdominal pain, pulsatile mass and gastrointestinal bleeding is an infrequent presentation and it is described in only $11 \%$ of patients [17-20].

\section{Diagnosis}

There are many modalities that may aid in the diagnosis of an AEF, but without strong clinical suspicion, these modalities may be futile. The initial evaluation of AEF should be started by esophagogastroduodenoscopy(EGD) to identify the cause of bleeding. The whole duodenum must be investigated in EGD because of the frequent location of the AEF in its 3rd and 4th parts. Findings suggestive of AEF on EGD include visible graft, bleeding, adherent clot, or ulcer or pulsatile mass (Figures $3 \& 4$ ). CT scan has $30-61 \%$ sensitivity in identifying PAEF, and 90\% sensitivity and specificity in identifying SAEF. In addition to identifying the location, it may aid in the identification of an infection or abscess formation if present. Signs found on CT imaging may be gas shadow in or around the graft (sensitivity $40 \%$ and specificity $100 \%$ ), focal wall inflammation and thickening, visible graft (sensitivity $22 \%$ and specificity $100 \%$ ), soft tissue collection around the aorta (specificity 92\% and sensitivity 90\%), swelling or hematoma around the graft, intravenous contrast within the GI lumen or around the aorta, loss of calcification or tear in the aortic wall (specificity $75 \%$ and sensitivity 89\%), pseudoaneurysm, and duodenal hematoma (Figures 5 \& 6). 


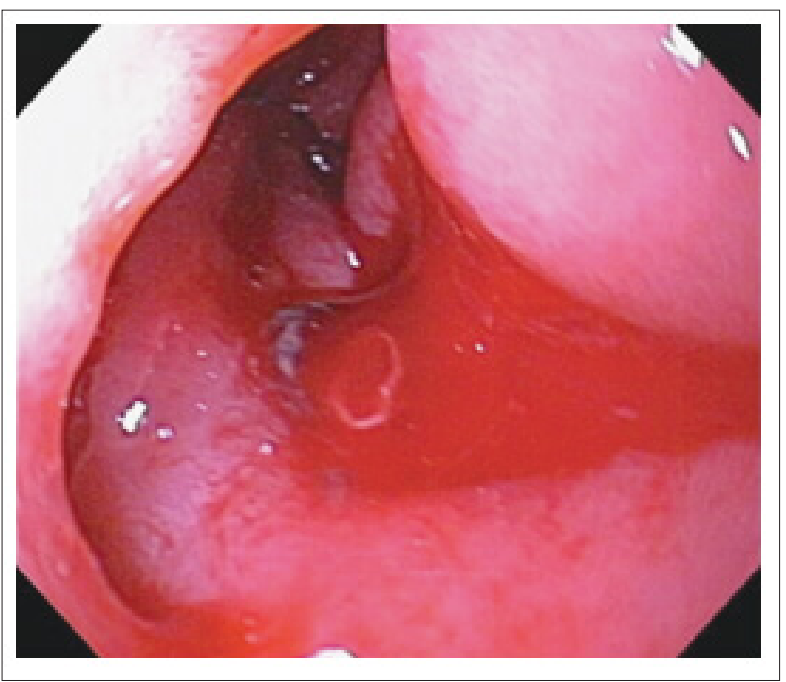

Figure 3: Visible graft with bleeding on (EGD)..

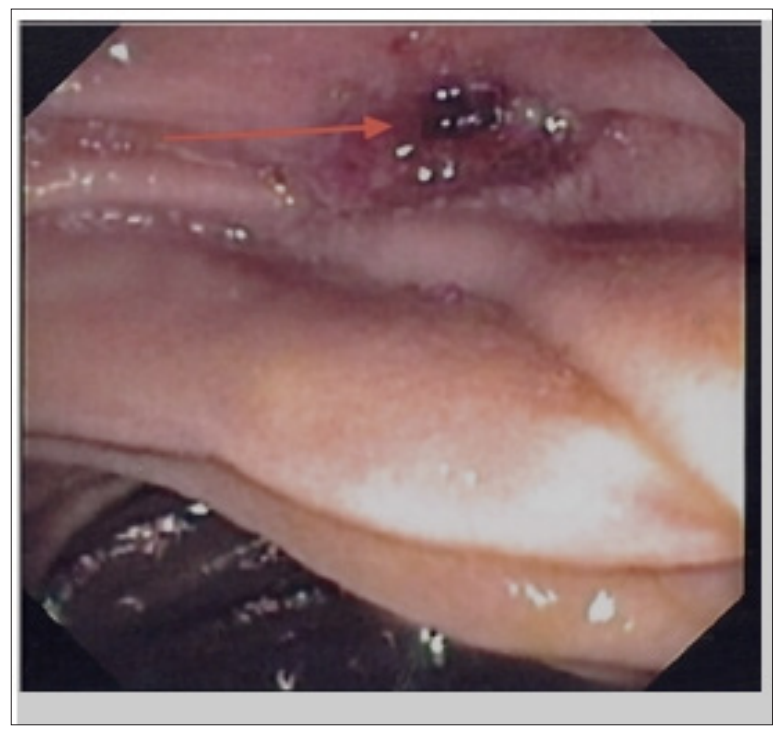

Figure 4: Push enteroscopy showing aortoenteric fistula with bleeding (arrow).

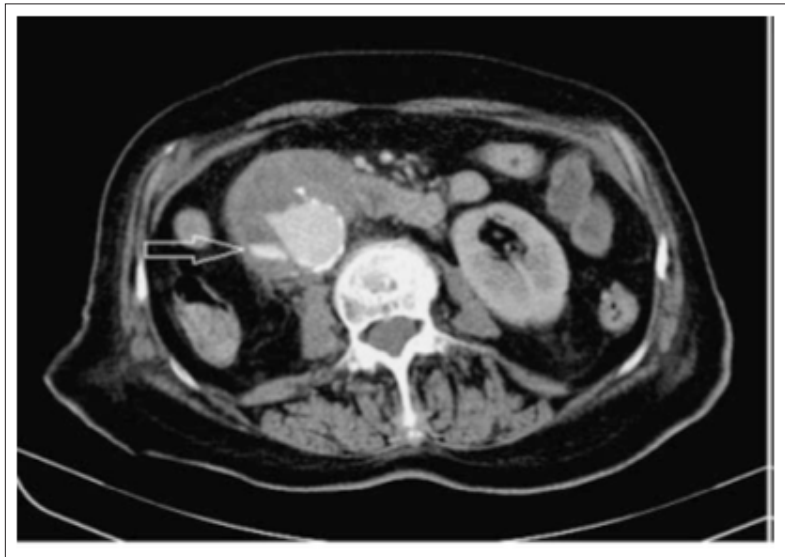

Figure 5: CECT: visible true lumen of the aneurysm with extravasation of contrast medium at the level of the second part of the duodenum (arrow).

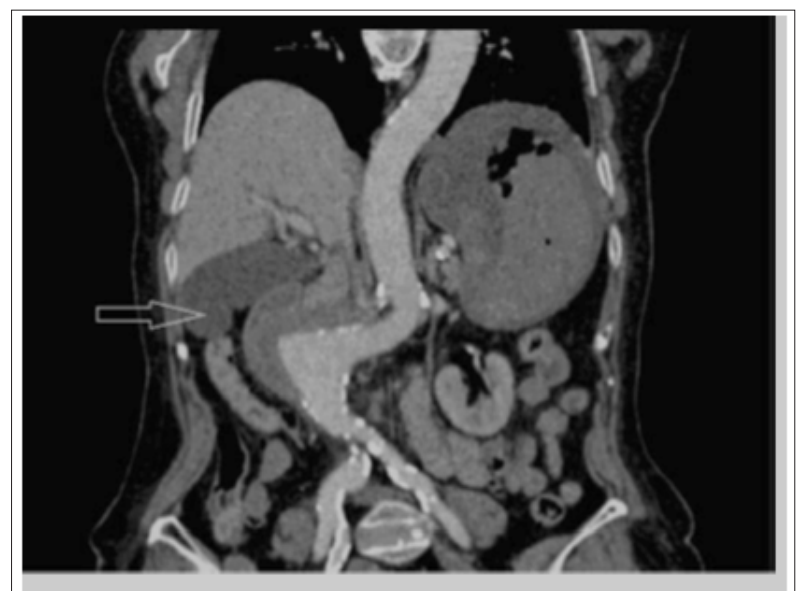

Figure 6: CT reconstruction: aneurysm with mass effect on the duodenum (arrow). Note the absence of the calcified atheroma on the right aortic contour that suggests aneurysmal rupture.

CT scan has $30-61 \%$ sensitivity in identifying PAEF, and $90 \%$ sensitivity and specificity in identifying SAEF. In addition to identifying the location, it may aid in the identification of an infection or abscess formation if present. Signs found on CT imaging may be gas shadow in or around the graft (sensitivity $40 \%$ and specificity 100\%), focal wall inflammation and thickening, visible graft (sensitivity 22\% and specificity 100\%), soft tissue collection around the aorta (specificity $92 \%$ and sensitivity 90\%), swelling or hematoma around the graft, intravenous contrast within the GI lumen or around the aorta, loss of calcification or tear in the aortic wall (specificity 75\% and sensitivity 89\%), pseudoaneurysm, and duodenal hematoma [21-23].

\section{Treatment}

Emergency surgery should be undertaken, as soon the diagnosis is evident or there is strong suspicion. The gold standard in the treatment of PAEF is surgery. At surgery, culture and debridement of the aneurysmal aorta are done. Aortic repair is done with a prosthetic graft, using Dacron Silver prosthesis, which is the preferred choice in a septic or potentially septic area; or with an extra-anatomic by-pass, or with the substitution of the aortic tract with a venous prosthesis (neoaortoiliac system, NAIS) or with the cryopreserved arterial homografts. And then a primary repair of the gastrointestinal tract is done in situations where there is no gross contamination of the peritoneal cavity. The endovascular aneurysm repair (EVAR) of the PAEF, as reported in the literature, could be applied in specific situations as a "bridge" treatment in patients with hemodynamic instability, where immediate surgery is contraindicated and has to be delayed. This solution is still controversial, especially for the persistence of the infective site and the risk of EVAR's infection.

Deshpande et al were the first to describe an endovascular repair of an SAEF in a high-risk patient. 6 The advantages of the endovascular approach are the rapid control of hemorrhaging and the avoidance of an intervention in a hostile abdomen or the avoidance of a procedure in patients with severe comorbidities 
unfit for open surgery. On contrary, the endovascular approach has great limitations as the bowel defect is not repaired; thus, infection, if present, may persist, and retroperitoneum debridement is not feasible. Recent review study on outcomes after the endovascular repair of SAEF by Kakkos et al showed that the endovascular approach was associated with a high incidence of persistent, or recurrent infection, or recurrent bleeding that significantly limited survival rates. Prognosis were worse in patients with perioperative sepsis, large bowel fistulization, and no intestinal repair. Bergqvist and Björck also reported that preoperative evidence of sepsis was an indicating factor for unfavorable outcomes. So endovascular management of the SAEF without further aortic reconstruction, followed by isolation of the eroded part of the duodenum and therapy, can be considered as a potentially effective treatment. Postoperatively, the patient should be treated with intravenous broad-spectrum antibiotics for up to 6 weeks according to the culture results (Figures 7 \& 8).

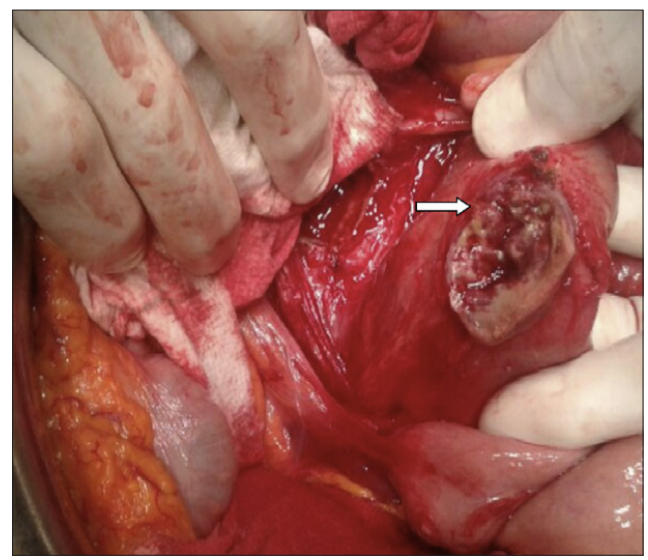

Figure 7: External view of the affected jejunum loop after en bloq resection of the aneurysmatic aortic defect was carried out.

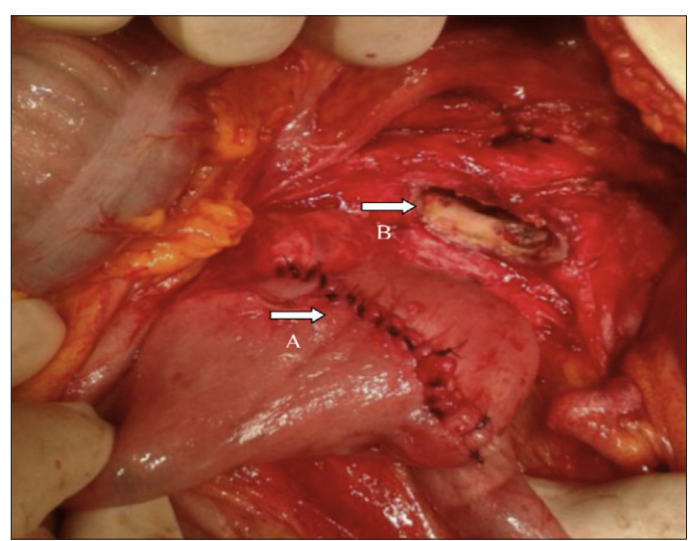

Figure 8: Prisssmary suture of the jejunal defect (A). The aneurysmatic aortic defect still remains opened in the picture, showing the intern fistulous orifice (B).

\section{Conclusion}

In conclusion, AEF is a very rare but often fatal condition. It should be suspected in cases of GI bleeding associated with aortic aneurysm. Diagnosis is usually not easy, but it will be crucial for the prognosis of these patients. The use of CT scan may assist in establishing an early diagnosis. Emergent surgical treatment is the best chance of survival of these patients. Endovascular approach in selected cases may ameliorate complications associated with surgery [24].

\section{References}

1. Jan G, Ferenc S, Magdalena K, Paprocka-Borowicz M, Piotr D, et al. (2016) Secondary aortoenteric fistula after abdominal aortic graft implementation in our own material. Adv Clin Exp Med 25(6): 12651271.

2. Sojun H, Koichi A, Masaya S, Toshihiro K, Hitoshi S (2016) Successful resolution of a hemorrhagic pancreatic pseudocyst ruptured into the stomach complicating obstructive pancreatitis due to pancreatic cancer: A case report. Hoshimoto World Journal of Surgical Oncology 14: 46.

3. Vernadakis S, Christodoulou E, Treckmann J, Saner F, Paul A, et al. (2009) Pseudoaneurysmal rupture of the common hepatic artery into the biliodigestive anastomosis. A Rare Cause of Gastrointestinal Bleeding. JOP 10(4): 441-444.

4. Chin CC, Yeh CY, Kuo YH, Wang JY (2008) Massive lower gastrointestinal bleeding from an external iliac artery fistula in a patient with bladder cancer. Chang Gung Med J 31(6): 612-615.

5. Nirmit D, Sagar P, Chinyere N, Lok S, Carl T, et al. (2013) Arteriojejunal fistula presenting with recurrent obscure gi hemorrhage in a patient with a failed pancreas allograft. Case Rep Transplant 2013: 4.

6. Senadhi V, Brown JC, Arora D, Shaffer R, Shetty D, et al. (2010) A mysterious cause of gastrointestinal bleeding disguising itself as diverticulosis and peptic ulcer disease: A review of diagnostic modalities for aortoenteric fistula. Case Rep Gastroenterol 4(3): 510-517.

7. Muñoz-Villafranca C, García-Kamirruaga Í, Góme-García P, Atín-delCampo V, Bárcena-Robredo V, et al. (2015) Pseudoaneurysm of the cystic artery: An uncommon cause of upper gastrointestinal bleeding in a case of xanthogranulomatous cholecystitis. Rev Esp Enferm Dig 107(6): 375376.

8. Lazaris AM, Tsapralis D, Patapis P, Mproutzos E, Tzathas H, et al. (2009) Aortoiliac endograft-enteric fistula due to an ingested toothpick. J Vasc Surg 50(3): 640-643.

9. Um SJ, Park BH, Son C (2009) An Aortoesophageal fistula in patient with lung cancer after chemo-irradiation and subsequent esophageal stent implantation. J Thorac Oncol 4(2): 263-265.

10. Karthaus EG, Post IC, Akkersdijk GJ (2016) Spontaneous aortoenteric fistula involving the sigmoid: A case report and review of literature. Int J Surg Case Rep 19: 97-99.

11. Pérez-Legaz J, Marín-Hargreaves G, Ramírez M, Moya P, Arroyo A (2013) Renal-appendicular fistula of the renal graft in a transplanted patient: an uncommon form of lower gastrointestinal hemorrhage. Cir Esp 91(6): 397-399.

12. Zhou JC, Xu QP, Shen LG, Pan KH, Mou YP (2009) Aortoduodenal fistula following aortic reconstruction of a pseudoaneurysm caused by stab wound 12 years ago. J Zhejiang Univ Sci B 10(5): 400-403.

13. Marek T, Hendryk V, Christian W, Reimer A (2014) Aortoenteric Fistula as a Complication of Open Reconstruction and Endovascular Repair of Abdominal Aorta. Radiol Res Pract 2014: 6.

14. Moulakakis KG, Kakisis J, Dalainas I, Liapis CD, Smyrniotis V (2015) Endovascular Management of Secondary Aortoduodenal Fistula: The Importance of Gut Restoration. Int J Angiol 24(1): 55-58.

15. Bas A, Simsek O, Kandemirli SG, Rafiee B, Gulsen F, et al. (2015) Evolution of Computed Tomography Findings in Secondary Aortoenteric Fistula. Iran J Radiol 12(2): e22759.

16. George E, Philippakisa, Marios M (2013) Surgical treatment of primary aortojejunal fistula. Int J Surg 4 (5): 477-479. 
17. Costea R, Vasiliu EC, Zărnescu NO, Neagu S (2015) Primary Aortoenteric Fistula: Case Report. Chirurgia (Bucur) 110(1): 78-80.

18. Shulik O, Marling K, Butler J (2013) Primary aorto-enteric fistula-A unique complication of poorly differentiated large B-cell lymphoma. Am J Case Rep 14: 194-197.

19. Guner A, Mentese U, Kece C, Kucuktulu U (2013) A rare and forgotten diagnosis of gastrointestinal bleeding: Primary aortoduodenal fistula. BMJ Case Rep 2013.

20. Ahmed S, Patel C, Schmitt C, Parajuli D (2016) A Deadly Connection: Aortoenteric Fistula as a Cause of Acute Upper Gastrointestinal Bleeding. ACG Case Reports J 3(3): 152-153.
21. Ho S, Liu B, Loya R, Koury I (2016) Primary aortoenteric fistula: A rare case of a massive gastrointestinal bleed. Cureus 8(9): e766.

22. Malik MU, Ucbilek E, Sherwal AS (2015) Critical gastrointestinal bleed due to secondary aortoenteric fistula. J Community Hosp Intern Med Perspect 5(6): 29677.

23. Varetto G, Gibello L, Trevisan A, Castagno C, Garneri P, et al. (2015) Primary Aortoenteric Fistula of a Saccular Aneurysm. Korean Circ J 45(4): 337-339.

24. Fernández de Sevilla E, Echeverri JA, Boqué M, Valverde S, Ortega N, et al. (2015) Life-threating upper gastrointestinal bleeding due to a primary aorto-jejunal fistula. Int J Surg Case Rep 8C: 25-28. 